\title{
Dose Trends of Aripiprazole from 2004 to 2014 in Psychiatric Inpatients in Korea
}

\author{
Young Sup Woo ${ }^{1}$, In Hee Shim ${ }^{2}$, Sang-Yeol Lee ${ }^{3}$, Dae-Bo Lee ${ }^{4}$, Moon-Doo Kim ${ }^{5}$, Young-Eun Jung ${ }^{5}$, Jonghun Lee ${ }^{6}$, \\ Seunghee Won ${ }^{7}$, Duk-In Jon ${ }^{8}$, Won-Myong Bahk ${ }^{1}$ \\ ${ }^{1}$ Department of Psychiatry, College of Medicine, The Catholic University of Korea, Seoul, ${ }^{2}$ Department of Psychiatry, Cancer Center, Dongnam \\ Institute of Radiological \& Medical Sciences, Busan, ${ }^{3}$ Department of Psychiatry, Wonkwang University Hospital, Wonkwang University School \\ of Medicine, Iksan, ${ }^{4}$ Department of Psychiatry, National Forensic Hospital, Gongju, ${ }^{5}$ Department of Psychiatry, Jeju National University School \\ of Medicine, Jeju, ${ }^{6}$ Department of Psychiatry, Catholic University of Daegu School of Medicine, Daegu, ${ }^{7}$ Department of Psychiatry, Kyungpook \\ National University Hospital, Kyungpook National University College of Medicine, Daegu, ${ }^{8}$ Department of Psychiatry, Hallym University College \\ of Medicine, Anyang, Korea
}

\begin{abstract}
Objective: Although aripiprazole has been widely used to treat various psychiatric disorders, little is known about the adequate dosage for Asian patients in clinical practice. Hence, we evaluated the initial and maximum doses of aripiprazole from 2004 to 2014 to estimate the appropriate dosage for Korean psychiatric inpatients in clinical practice.

Methods: In this retrospective study, we reviewed the medical records of patients who were hospitalized in five university hospitals in Korea from March 2004 to December 2014. The psychiatric diagnosis according to the text revision of the Diagnostic and Statistical Manual of Mental Disorders, 4th edition during index hospitalization and the initial and maximum doses of aripiprazole were evaluated.

Results: There were 74 patients in Wave 1 (2004-2006), 201 patients in Wave 2 (2007-2010), and 353 patients in Wave 3 (2011-2014). The initial doses of aripiprazole in all diagnostic groups were significantly lower in Wave 3 than in Wave 2. The maximum doses of aripiprazole in each diagnostic group were not significantly different among Waves 1, 2 , and 3.

Conclusion: The relatively low initial doses of aripiprazole documented in our study may reflect a strategy by clinicians to minimize the side effects associated with aripiprazole use, such as akathisia.
\end{abstract}

KEY WORDS: Aripiprazole; Prescribing pattern; Initial dose.

\section{INTRODUCTION}

Aripiprazole is regarded as a third-generation antipsychotic due to its unique pharmacological profile. ${ }^{1)}$ Its distinct pharmacological profile suggests aripiprazole may be effective in treating various psychiatric disorders. ${ }^{2-6)}$ Moreover, the safety profile of aripiprazole, with low risk of weight gain, sedation and hyperprolactinemia, as well as its relatively high cardiovascular safety in comparison with other atypical antipsychotics, ${ }^{7)}$ may also increase the clinical utility of aripiprazole.

One of the key issues in clinical practice is the choice of antipsychotic dose because it can determine the efficacy

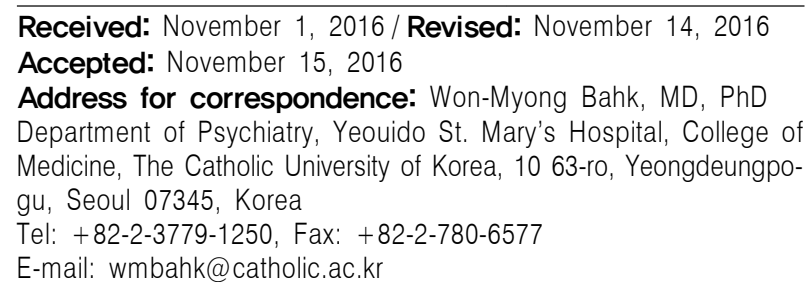

and safety/tolerability of treatment. However, published evidence from studies assessing the use of atypical antipsychotics in psychiatric practice show that prescribed doses can diverge from those recommended on the product label. ${ }^{8,9)}$ In real world situations, clinicians may modify their prescribing practices, with some antipsychotics being prescribed at much lower or at much higher doses than originally anticipated, after gaining clinical experience with new medications. ${ }^{10)}$ Because some side effects such as agitation, akathisia and extrapyramidal symptoms may limit its clinical use, ${ }^{11)}$ and the overall safety and tolerability of aripiprazole is favorable compared to other psychiatric medications, it would be regarded as prudent to aim for the minimum effective dose. However, in spite of attempts to establish a dose-response range for aripiprazole, ${ }^{1,12)}$ little is known about the adequate dosage in clinical practice for Asian patients with various psychiatric disorders.

The purpose of the present study was to evaluate the initial and maximum doses of aripiprazole over a decade to

() This is an Open-Access article distributed under the terms of the Creative Commons Attribution Non-Commercial License (http://creativecommons.org/licenses/by-nc/4.0) which permits unrestricted non-commercial use, distribution, and reproduction in any medium, provided the original work is properly cited. 
estimate appropriate dosage in clinical practice. We hypothesized that there was a measurable change in dosing patterns during 2004-2014 in Korean psychiatric inpatients.

\section{METHODS}

In this retrospective study, we reviewed the medical records of patients who were hospitalized in the psychiatric ward of five university hospitals in Korea from March 2004 to December 2014. The patients were at least 18 years of age, prescribed aripiprazole during the index hospitalization and were given at least one prescription for oral aripiprazole. Patients who were treated with aripiprazole for a psychiatric disorder before the index hospitalization, who had severe medical conditions which would affect aripiprazole prescription, and were admitted for reasons other than treatment of psychiatric symptoms during index hospitalization, including for social reasons or diagnostic purposes, were excluded.

The psychiatric diagnosis according to the text revision of the Diagnostic and Statistical Manual of Mental Disorders, 4th edition (DSM-IV-TR) during index hospitalization and the initial and maximum doses of aripiprazole were evaluated. We also reviewed the demographic and clinical data, including age, sex, marriage, employment, years of education and concomitant medications.

We compared baseline demographic variables among Waves 1 (2004-2006), 2 (2007-2010) and 3 (2011-2014) using univariate one-way analysis of variance (ANOVA) with Bonferroni correction for continuous variables and a chi-square test for categorical variables. Analysis of covariance (ANCOVA) with Bonferroni post-hoc pair-wise comparisons, adjusting for the statistically significant variables from univariate analysis including age and concomitant medications, was performed to compare the initial and maximum doses of aripiprazole for each diagnostic group among waves. A $p$ value $<0.05$ ( $p<0.017$ with Bonferroni's correction) was considered to indicate statistical significance.

This study was approved by the institutional review board of each hospital, and was conducted according to the Declaration of Helsinki. The institutional review board approved the exemption for informed consent because this was a retrospective chart review study.

\section{RESULTS}

A total of 628 patients were included in this study. There were 74 patients in Wave 1, 201 patients in Wave 2, and 353 patients in Wave 3. A detailed overview of the demographic and clinical characteristics of the patients is presented in Table 1. There was a significant difference in mean age among waves $(p=0.012)$. The mean age of Wave $1(34.0 \pm 10.4)$ was significantly different from that of Wave $2(39.9 \pm 15.7, p=0.008)$ and Wave $3(40.1 \pm 17.5$, $p=0.003)$.

Table 1. Comparisons of baseline demographic and clinical characteristics among Wave 1 (2004-2006), Wave 2 (2007-2010) and Wave 3 (2011-2014)

\begin{tabular}{|c|c|c|c|c|c|c|c|c|}
\hline & $\begin{array}{l}\text { Full sample } \\
\qquad(n=628)\end{array}$ & $\begin{array}{l}\text { Wave } 1 \\
(n=74)\end{array}$ & $\begin{array}{l}\text { Wave } 2 \\
(n=201)\end{array}$ & $\begin{array}{l}\text { Wave } 3 \\
(n=353)\end{array}$ & 1 vs. 2 vs. 3 & 1 vs. 2 & 1 vs. 3 & 2 vs. 3 \\
\hline Age (yr) & $39.3 \pm 16.4$ & $34.0 \pm 10.4$ & $39.9 \pm 15.7$ & $40.1 \pm 17.5$ & 0.012 & $0.008^{*}$ & $0.003^{*}$ & 0.849 \\
\hline Male & $252(40.1)$ & $34(45.9)$ & $81(40.3)$ & $137(38.8)$ & 0.522 & 0.400 & 0.255 & 0.730 \\
\hline Married & $261(41.6)$ & $23(31.1)$ & $88(43.8)$ & $150(42.6)$ & 0.141 & 0.057 & 0.069 & 0.768 \\
\hline Employed & $334(53.2)$ & $35(47.3)$ & $106(52.7)$ & $193(54.8)$ & 0.490 & 0.424 & 0.247 & 0.660 \\
\hline Education (yr) & $13.0 \pm 3.8$ & $13.7 \pm 2.7$ & $13.2 \pm 3.2$ & $12.7 \pm 4.2$ & 0.092 & 0.309 & 0.045 & 0.180 \\
\hline \multicolumn{9}{|c|}{ Concomitant medication } \\
\hline Other AAPs & $204(32.5)$ & $20(27.0)$ & $55(27.4)$ & $129(36.5)$ & 0.048 & 0.956 & 0.118 & 0.027 \\
\hline MSs & $233(37.1)$ & $14(18.9)$ & $78(38.8)$ & $141(39.9)$ & 0.003 & $0.002^{*}$ & $0.001^{*}$ & 0.792 \\
\hline ADs & $239(38.1)$ & 31 (41.9) & $57(28.4)$ & $151(42.8)$ & 0.003 & 0.033 & 0.889 & $0.001^{*}$ \\
\hline \multicolumn{9}{|l|}{ Diagnosis } \\
\hline SPR & $290(46.2)$ & $57(77.0)$ & $96(47.8)$ & $137(38.8)$ & $<0.001$ & $<0.001^{*}$ & $<0.001^{*}$ & 0.040 \\
\hline MDD & $138(22.0)$ & $7(9.5)$ & $41(20.4)$ & $90(25.5)$ & 0.008 & 0.034 & $0.003^{*}$ & 0.175 \\
\hline BP-M & $116(18.5)$ & $7(9.5)$ & $45(22.4)$ & $64(18.1)$ & 0.048 & $0.015^{\star}$ & 0.069 & 0.225 \\
\hline BP-D & $41(6.5)$ & $1(1.4)$ & $10(5.0)$ & $30(8.5)$ & 0.043 & 0.174 & 0.031 & 0.123 \\
\hline Others & $43(6.8)$ & $2(2.7)$ & $9(4.5)$ & $32(9.1)$ & 0.039 & 0.505 & 0.066 & 0.047 \\
\hline
\end{tabular}

Values are presented as mean \pm standard deviation or number (\%).

AAPs, atypical antipsychotics; MSs, mood stabilizers; Ads, antidepressants; SPR, schizophrenia and other psychotic disorders; MDD, major depressive disorders and other depressive disorders; BP-M, bipolar disorder manic/mixed/hypomanic episode; BP-D, bipolar depressive episode.

*Bonferroni corrected significance of $p<0.017$ in the post hoc analysis. 
The use of concomitant medications with aripiprazole was significantly different among waves, as well. The use of other atypical antipsychotics in Wave 1 was $27.0 \%$ $(\mathrm{n}=20)$ and $27.4 \%(\mathrm{n}=55)$ in Wave 2 and increased to $36.5 \%(n=129)$ in Wave 3, but the difference between Waves 1 and $3(p=0.118)$ and 2 and $3(p=0.027)$ did not reach statistical significance after Bonferroni's correction. However, the concomitant use of mood stabilizers/anticonvulsants with aripiprazole was significantly different between Waves $1(18.9 \%, \mathrm{n}=14)$ and $2(38.8 \%, \mathrm{n}=78$, $p=0.002)$, and 1 and $3(39.9 \%, \mathrm{n}=141, p=0.001)$. For antidepressants, $28.4 \%$ of patients in Wave $2(n=57)$ and $42.8 \%(n=151)$ of patients in Wave 3 were prescribed antidepressants, which constituted a significant difference $(p=0.001)$.

In total, the initial dose of aripiprazole was significantly lower in Wave $3(7.0 \pm 3.9 \mathrm{mg} /$ day $)$ when compared to Waves $1(10.9 \pm 4.6 \mathrm{mg} / \mathrm{day}, p<0.001)$ and $2(10.7 \pm 5.6$ $\mathrm{mg} /$ day, $p<0.001)$. The initial doses of aripiprazole in all diagnostic groups were significantly lower in Wave 3 than in Wave 2 (Table 2). When comparing the initial doses of aripiprazole between Waves 3 and 1 in each diagnostic group, there was a significant difference in schizophrenia and other psychotics disorders $(11.0 \pm 4.1 \mathrm{mg}$ /day in Wave 1 , $8.2 \pm 3.8 \mathrm{mg} /$ day in Wave $3, p<0.001)$. The maximum doses of aripiprazole in all subjects and for each diagnostic group were not significantly different among Waves 1,2 , and 3 .

\section{DISCUSSION}

The mean initial dose of aripiprazole in Wave 3 was
$7.0 \pm 3.9 \mathrm{mg} /$ day for schizophrenic patients and $8.6 \pm 4.1$ $\mathrm{mg}$ /day for bipolar manic/mixed patients which was lower than the recommended dose (10-15 mg/day for schizophrenia and $15 \mathrm{mg} /$ day for bipolar manic/mixed episode) according to the product information sheet. For the treatment of major depressive disorders, $5.0 \pm 3.3 \mathrm{mg} /$ day of aripiprazole was prescribed initially in Wave 3, which was in accordance with the product information sheet (2-5 $\mathrm{mg}$ /day). The results from the present study show that the initial doses of aripiprazole, and not the maximum doses, decreased in hospitalized psychiatric patients with the accumulation of clinical experience in aripiprazole use. The relatively low initial doses of aripiprazole documented in our study may reflect a strategy by clinicians to minimize the side effects associated with aripiprazole use. One of the most common adverse effects associated with aripiprazole is akathisia which can adversely affect patients' adherence to medication, potentially leading to reluctance among clinicians in prescribing the drug. ${ }^{11,13,14)}$ In a pooled analysis of short-term aripiprazole clinical trials, the incidence of akathisia was $9.0 \%$ in schizophrenia trials, and $17.9 \%$ in bipolar trials. ${ }^{15)}$ Although a study analyzing safety and tolerability data from short-term trials with aripiprazole in schizophrenia reported that there was no dose-response relationship with aripiprazole for akathisia, ${ }^{16)}$ a few studies suggested an association between akathisia and dosing of antipsychotics including aripiprazole. Miller et al ${ }^{17)}$ reported that higher doses or a rapid dose increase of antipsychotics could be a possible risk factor for akathisia. Moreover, Basu and Brar ${ }^{18)}$ suggested that starting aripiprazole at lower doses, either 5 or

Table 2. Comparisons of starting and maximum dose of aripiprazole among Wave 1 (2004-2006), Wave 2 (2007-2010), and Wave 3 (2011-2014)

\begin{tabular}{|c|c|c|c|c|c|c|c|c|}
\hline \multirow{2}{*}{ Variable } & \multirow{2}{*}{$\begin{array}{l}\text { Aripiprazole dose } \\
\text { (mg/day) }\end{array}$} & \multirow{2}{*}{ Wave 1} & \multirow{2}{*}{ Wave 2} & \multirow{2}{*}{ Wave 3} & \multicolumn{4}{|c|}{ Covariate adjusted $p$ value } \\
\hline & & & & & 1 vs. 2 vs. 3 & 1 vs. 2 & 1 vs. 3 & 2 vs. 3 \\
\hline \multirow[t]{2}{*}{ Full sample } & Initial & $10.9 \pm 4.6$ & $10.7 \pm 5.6$ & $7.0 \pm 3.9$ & $<0.001^{*}$ & $>0.999$ & $<0.001^{*}$ & $<0.001^{*}$ \\
\hline & Maximum & $21.2 \pm 8.4$ & $20.3 \pm 9.3$ & $18.1 \pm 10.5$ & 0.142 & $>0.999$ & 0.368 & 0.325 \\
\hline \multirow[t]{2}{*}{ SPR } & Initial & $11.0 \pm 4.1$ & $11.9 \pm 5.8$ & $8.2 \pm 3.8$ & $<0.001^{*}$ & $>0.999$ & $<0.001^{*}$ & $<0.001^{*}$ \\
\hline & Maximum & $22.3 \pm 8.0$ & $23.4 \pm 8.0$ & $23.3 \pm 9.8$ & 0.990 & $>0.999$ & $>0.999$ & $>0.999$ \\
\hline \multirow[t]{2}{*}{ MDD } & Initial & $7.6 \pm 2.5$ & $6.8 \pm 3.8$ & $5.0 \pm 3.3$ & $0.004^{*}$ & $>0.999$ & 0.243 & $0.006^{*}$ \\
\hline & Maximum & $13.6 \pm 5.6$ & $12.3 \pm 6.8$ & $10.9 \pm 9.1$ & 0.297 & $>0.999$ & $>0.999$ & 0.419 \\
\hline \multirow[t]{2}{*}{ BP-M } & Initial & $12.9 \pm 8.6$ & $12.1 \pm 5.0$ & $8.6 \pm 4.1$ & $0.001^{*}$ & $>0.999$ & 0.048 & $0.003^{*}$ \\
\hline & Maximum & $22.1 \pm 9.1$ & $22.9 \pm 8.6$ & $20.7 \pm 7.6$ & 0.459 & $>0.999$ & $>0.999$ & 0.651 \\
\hline \multirow[t]{2}{*}{ BP-D } & Initial & 15 & $10.5 \pm 4.4$ & $6.3 \pm 2.9$ & $0.004^{*}$ & 0.633 & 0.066 & $0.018^{*}$ \\
\hline & Maximum & 30 & $21.0 \pm 10.2$ & $16.8 \pm 8.5$ & 0.261 & $>0.999$ & 0.537 & 0.814 \\
\hline \multirow[t]{2}{*}{ Other diagnosis } & Initial & $10.0 \pm 0.0$ & $8.3 \pm .2$ & $4.8 \pm 2.8$ & $0.005^{*}$ & $>0.999$ & 0.180 & $0.009^{*}$ \\
\hline & Maximum & $10.0 \pm 0.0$ & $9.7 \pm 5.1$ & $12.0 \pm 9.5$ & 0.631 & $>0.999$ & $>0.999$ & $>0.999$ \\
\hline
\end{tabular}

Values are presented as mean \pm standard deviation.

SPR, schizophrenia and other psychotic disorders; MDD, major depressive disorders and other depressive disorders; BP-M, bipolar disorder manic/mixed/hypomanic episode; BP-D, bipolar depressive episode.

*Bonferroni corrected significance of $p<0.017$ in the post hoc analysis. 
$10 \mathrm{mg} /$ day, minimizes the risk of akathisia and that starting at doses greater than $30 \mathrm{mg} /$ day increases the risk of schizoaffective disorder. In line with the suggestion by Nelson et al. ${ }^{19)}$ that lowering the starting dose of aripiprazole represents one of the most widely used strategies to minimize the incidence or the severity of akathisia, the results from the present study may reflect clinical experience that a low initial dose could reduce the risk of akathisia.

The principal limitation here was the retrospective nature of this work and the relatively small sample size taken from five university hospitals in Korea. Thus, we cannot address the explicit reasons for choosing particular medications and doses with the limited generalizability to other populations. Second, we collected only initial and maximum doses of aripiprazole as a representative value for dosing patterns, and the mean or modal dose was not evaluated. Finally, psychiatric and medical comorbidities in subjects which might affect the prescribing and dosing patterns in the present study were not evaluated. Despite these limitations, this study has strength in that it shows long-term trends in prescribing patterns of aripiprazole. Although "prescribing-based evidence" is not a substitute for "evidence-based prescribing,", it would be worthy to update dose trend information through controlled trials in order to test the utility of different dose strategies, as fixed-dose studies of aripiprazole that clearly elucidate the dose-response relationship with effectiveness/tolerability are generally lacking.

We found that the initial doses of aripiprazole in all diagnostic groups were significantly lower in 2011-2014 than in 2007-2010. However, there were no significant changes over time in the maximum doses of aripiprazole in each diagnostic group. Although it is not surprising since there are reports indicating that the higher initial doses can provoke more adverse events, this study could be meaningful as we were able to confirm that the evidences are reflected to real clinical practice.

\section{REFERENCES}

1. Di Sciascio G, Riva MA. Aripiprazole: from pharmacological profile to clinical use. Neuropsychiatr Dis Treat 2015;11:2635-2647.

2. Stip E, Tourjman V. Aripiprazole in schizophrenia and schizoaffective disorder: a review. Clin Ther 2010;32 Suppl 1:S3-S20.

3. Moro MF, Carta MG. Evaluating aripiprazole as a potential bipolar disorder therapy for adults. Expert Opin Investig Drugs 2014;23:1713-1730.

4. Pae CU, Forbes A, Patkar AA. Aripiprazole as adjunctive therapy for patients with major depressive disorder: overview and implications of clinical trial data. CNS Drugs 2011;25:109-127.

5. Farmer CA, Aman MG. Aripiprazole for the treatment of irritability associated with autism. Expert Opin Pharmacother 2011;12:635-640.

6. Zheng W, Li XB, Xiang YQ, Zhong BL, Chiu HF, Ungvari GS, et al. Aripiprazole for Tourette's syndrome: a systematic review and meta-analysis. Hum Psychopharmacol 2016;31:11-18.

7. Khanna P, Suo T, Komossa K, Ma H, Rummel-Kluge C, El-Sayeh HG, et al. Aripiprazole versus other atypical antipsychotics for schizophrenia. Cochrane Database Syst Rev 2014;(1):CD006569.

8. Hartung DM, Wisdom JP, Pollack DA, Hamer AM, Haxby DG, Middleton L, et al. Patterns of atypical antipsychotic subtherapeutic dosing among Oregon Medicaid patients. $J$ Clin Psychiatry 2008;69:1540-1547.

9. Citrome L, Reist C, Palmer L, Montejano L, Lenhart G, Cuffel $\mathrm{B}$, et al. Dose trends for second-generation antipsychotic treatment of schizophrenia and bipolar disorder. Schizophr Res 2009;108:238-244.

10. Citrome L, Jaffe A, Levine J. Dosing of second-generation antipsychotic medication in a state hospital system. J Clin Psychopharmacol 2005;25:388-391.

11. Pae CU. A review of the safety and tolerability of aripiprazole. Expert Opin Drug Saf 2009;8:373-386.

12. Mace S, Taylor D. Aripiprazole: dose-response relationship in schizophrenia and schizoaffective disorder. CNS Drugs 2009;23:773-780.

13. Kane JM. Extrapyramidal side effects are unacceptable. Eur Neuropsychopharmacol 2001;11 Suppl 4:S397-S403.

14. Ghaemi SN, Hsu DJ, Rosenquist KJ, Pardo TB, Goodwin FK. Extrapyramidal side effects with atypical neuroleptics in bipolar disorder. Prog Neuropsychopharmacol Biol Psychiatry 2006;30:209-213.

15. Kane JM, Barnes TR, Correll CU, Sachs G, Buckley P, Eudicone $\mathrm{J}$, et al. Evaluation of akathisia in patients with schizophrenia, schizoaffective disorder, or bipolar I disorder: a post hoc analysis of pooled data from short-and long-term aripiprazole trials. J Psychopharmacol 2010;24:1019-1029.

16. Marder SR, McQuade RD, Stock E, Kaplita S, Marcus R, Safferman AZ, et al. Aripiprazole in the treatment of schizophrenia: safety and tolerability in short-term, placebocontrolled trials. Schizophr Res 2003;61:123-136.

17. Miller CH, Hummer M, Oberbauer H, Kurzthaler I, DeCol C, Fleischhacker WW. Risk factors for the development of neuroleptic induced akathisia. Eur Neuropsychopharmacol 1997; 7:51-55.

18. Basu R, Brar JS. Dose-dependent rapid-onset akathisia with aripiprazole in patients with schizoaffective disorder. Neuropsychiatr Dis Treat 2006;2:241-243.

19. Nelson JC, Thase ME, Trivedi MH, Fava M, Han J, Van Tran Q, et al. Safety and tolerability of adjunctive aripiprazole in major depressive disorder: A pooled post hoc analysis (studies CN138-139 and CN138-163). Prim Care Companion J Clin Psychiatry 2009;11:344-352. 